\title{
Interstitiallösungen der Sedimente, Nährstoffgehalt des Wassers und Primärproduktion des Phytoplanktons in Seen
}

\author{
Waldemar OHLE \\ Hydrobiologische Anstalt der Max-Planck-Gesellschaft, Plön, Holstein
}

\begin{abstract}
Interstitial fluide of sediments, nutrition content of the water and primary production of phytoplankton in lakes. In four lakes of northern Germany (Schöhsee, Schluënsee, Plußsee and Großer Plöner See) the intensities of photosynthesis are significantly different because of their nutrient reserves, especially in phosphates and nitrates. The nutritional reservoir is represented by the interstitial fluids of the mud; there exists a direct relation between their contents of total phosphorus and ammonium ions and the primary production (phytoplankton $/ \mathrm{m}^{3} \times 24 \mathrm{hrs}$ ) in the euphotic zones. During the summer (1962) the rate of primary production in the four lakes is reduced as phosphates and nitrates are depleted in the epilimnion. During the discontinuity period (summer 1962) a vertical zonation in the intensity of primary production is maintained with a maximum in the lower epilimnion. This vertical gradient is due to (1) adaptation of algae (especially Oscillatoria and Anabaena species) to low illumination intensities, (2) favorable supply with nutrients.
\end{abstract}

\section{EINLEITUNG}

Die Primärproduktion des Phytoplanktons unterliegt im Meere wie in Binnengewässern einer Reihe von Faktoren, von denen die Nährstoffversorgung und das Licht, aber auch die Sinkgeschwindigkeit der Planktonorganismen beziehungsweise die Turbulenzströmungen des Wassers hervorragende Bedeutung haben. Die Beziehungen treten bei thermischer Schichtung der Gewässer besonders deutlich hervor, da sich zwischen der Oberschicht und der Unterschicht oder am Metalimnion eine Grenzzone mit hohen thermischen Gradienten und entsprechend ausgeprägtem Verweilen von Partikeln ausbildet. Hier reichern sich daher Detritusteilchen, mögen sie aus oberen Wasserzonen abgesunken sein oder durch Laminarströmungen herangeführt werden, ebenso wie lebende Planktonorganismen an. Aber die Photosynthese kann weiterhin erfolgen, sofern genügend Lichtenergie zur Verfügung steht. Die Vertikalausbreitung der Primärproduktion weicht dann scheinbar von ihrer direkten Gebundenheit an die Beleuchtungsintensitäten infolge unterschiedlicher Planktondichten $a b$.

Ein Parallellaufen der mit zunehmender Gewässertiefe abfallenden Lichtintensität und Photosyntheseaktivität wird außerdem durch überoptimale Lichtmengen unter-

${ }^{1}$ Herrn Professor Dr. W. Schäperclaus, Berlin-Friedrichshagen, zu seinem 65. Geburtstag gewidmet. 
bunden. Es handelt sich um eine unmittelbare Lichtschädigung der Algen (STEEMANN Nrelsen 1952), so daß das Maximum der Photosynthese nicht an der Gewässeroberfläche, sondern tiefer liegt (STEEmann Nielsen \& Jensen 1957, Ryther 1956, Rodhe 1958). Eine wesentliche Rolle spielt dabei die Lichtadaptation der Phytoplanktonarten (Steemann Nielsen \& Jensen 1. c., Ruther \& Menzel 1959, Steemann Nielsen \& Hansen 1961).

Bei der synoptischen produktionsbiologischen Untersuchung einer Reihe von Binnenseen, deren Epilimnion, beurteilt nach der Temperatur und den chemischen Eigenschaften, sich in starker Turbulenz befand, trafen wir auf ähnliche konvexe Verteilungskurven der Photosyntheseaktivität weit häufiger als ursprünglich erwartet. Es soll im folgenden die ausschlaggebende Wirkung der Nährstoffkonzentration des Wassers und vor allem des autochthonen Nährstoffnachschubes auf die Primärproduktion des Phytoplanktons sowie auf die Ausbildung ihrer Vertikalzonierung behandelt werden.

\section{METHODIK}

Die Interstitiallösungen wurden durch Zentrifugierung bei $3000 \mathrm{U} / \mathrm{min}$ und anschließend bei $17000 \mathrm{U} / \mathrm{min}$ der mit einem Bodengreifer aus den Seen entnommenen Sedimente gewonnen und ebenso wie die Wasserproben nach üblichen chemischen Methoden analysiert. Die Nitratbestimmung erfolgte nach Reduktion zu Nitrit (MuLLIN \& Riley 1955). Die Humusfarbe (Hufa) der Wässer wurde nach Alkalisierung kolorimetrisch durch Vergleich mit einer Methylorangelösung ermittelt und in Farbeinheiten (je 0,01 mg Methylorange/l) angegeben. Der Gehalt der Sedimente an Huminsäure wurde nach Eichung mit "Huminsäure МЕRск“ jedoch in $\mathrm{mg} / \mathrm{l}$ Huminsäure in die Tabelle aufgenommen. Zur Elementaranalyse der organischen Substanz diente die trockene Verbrennung im Sauerstoffstrom mit Hilfe der Herafus-Apparatur. Für die Sedimentuntersuchung wurden vorwiegend die von UNGEMACH (1960) dargestellten Methoden angewandt.

Die Netto-Produktion des Phytoplanktons wurde nach Markierung in etwa $120 \mathrm{ml}$ fassenden Glasflaschen bei 24stündiger Exposition „in situ" beziehungsweise „in vitro et loco“, also in den Wasserzonen, aus denen die einzelnen Wasserproben entnommen waren, bestimmt, und zwar nach Filtration durch Membranfilter (Gruppe 3) mit dem Methan-Durchflußzähler und Meßgerät FH 49 einschließlich automatischem Probenwechsler und Zeitdrucker der Firma Frieseke und HöpfNER, außerdem „in vitro" bei 4stïndiger Exposition im Rotationslichtthermostaten mit $8 \mathrm{U} / \mathrm{min}$ und durchschnittlicher Beleuchtung von 4400 Lux. Die Temperatur des Wasserbades wurde auf die in der Produktionszone des Sees herrschende Mitteltemperatur eingestellt.

\section{DIE PRIMARPRODUKTION DER SEEN IN BEZIEHUNG ZUM NAHRSTOFFRESERVOIR}

Wie GesSNER (1959) im zweiten Band seiner „Hydrobotanik“ klar umrissen hat und sich aus den hierfür berücksichtigten Veröffentlichungen der marinen und lim- 
nischen Biologie ergibt, übt die Beleuchtungsintensität neben den Nährstoffen, vor allem Phosphor und Stickstoff, erwartungsgemäß entscheidenden Einfluß auf die photosynthetische Leistung des Phytoplanktons aus. Aud bei gleichmäßiger Verteilung der Nährstoffe sowie des Planktons in der euphotischen Zone der Gewässer bildet sich eine Optimalregion der Photosynthese aus, wenn sie infolge starker Lichteinstrahlung in den obersten Wasserschichten eine Depression erleidet. Charakteristische Verteilungskurven dieser Art wurden unter anderem von SteEmann Nielsen \& JENSEN (1957) sowie von Ryther $(1956,1959)$ für die Meere und von RodHe et al. (1958) für den See Erken und andere Gewässer beschrieben. Ahnlich wie bei Landpflanzen sind jedoch

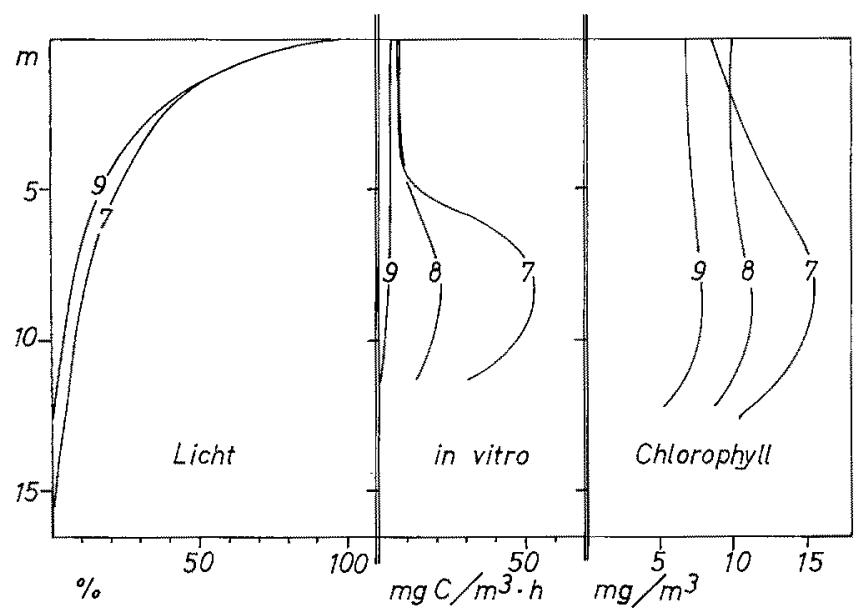

Abb. 1 B: Durchschnittliche Lichtyerteilung, Photosyntheseaktivität „in vitro" im Rotationslichthermostaten und Chlorophyllmengen in den verschiedenen Gewässertiefen des Schöhsees 1962

auch bei den Algen die Verhältnisse viel komplizierter, da ihre Lichtadaptation in den verschiedenen Gewässertiefen sowohl wie im Laufe des Jahres, wenigstens in nördlichen geographischen Breiten, die Relation Licht : Photosynthese erheblich verändert (TALling 1957, Rodhe et al. 1958, Stremann Nielsen \& Hansen 1959, Ryther 1959, Steemann Nielsen \& Hansen 1961). Besondere Bedeutung kann auch die Temperatur als Faktor der Photosyntheseaktivität erlangen, wie TALling (1957) für Asterionella und andere Diatomeen nachgewiesen hat. Die photosynthetische Leistung dieser Organismen erhöht sich bei einer Temperatursteigerung von $10^{\circ} \mathrm{C}$ um mehr als $100 \%$.

Selbstverständlich sind diese Befunde nicht unmittelbar zu verallgemeinern; denn die physiologischen Ansprüche der einzelnen Planktonarten hinsichtlich Licht, Temperatur und Nährstoffen sind unterschiedlich und bedürfen in den meisten Fällen noch eingehender experimenteller Arbeiten. Dennoch lassen sich aus der Photosyntheseaktivität der Planktonpopulationen und ihrer Vertikalzonierung in den Gewässern bereits wesentliche Schlußfolgerungen auf die wirksamen Faktoren ziehen.

Unsere Untersuchungen wurden im Laufe mehrerer Jahre an einigen holsteinischen Seen durchgeführt, und wir beziehen uns im folgenden auf die 1962 vom Schöhsee, Schluënsee, Plußsee und Großen Plöner See erhaltenen Ergebnisse. Diese Gewässer 


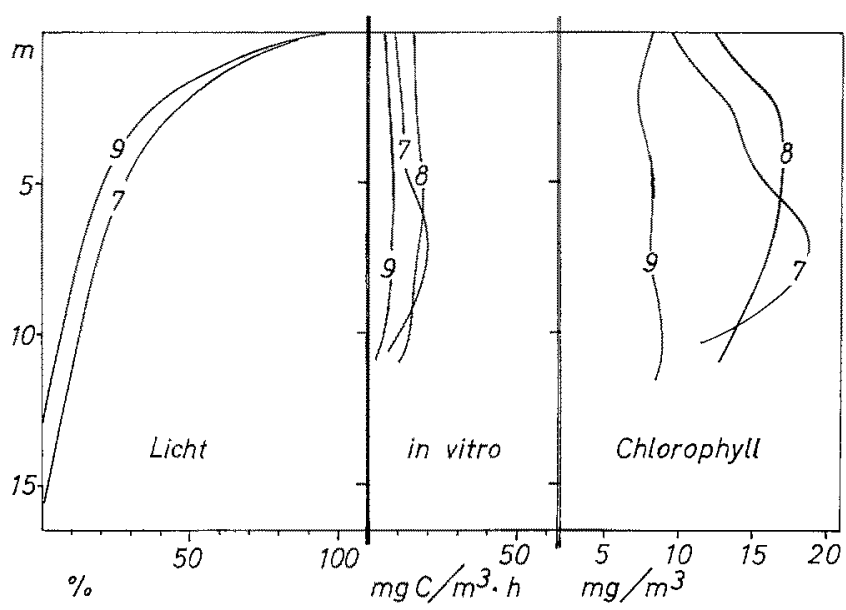

Abb. 2B: Durchschnittliche Lichtverteilung, Photosyntheseaktivität „in vitro" im Rotationslichtthermostaten und Chlorophyllmengen in den verschiedenen Gewässertiefen des Schluënsees 1962

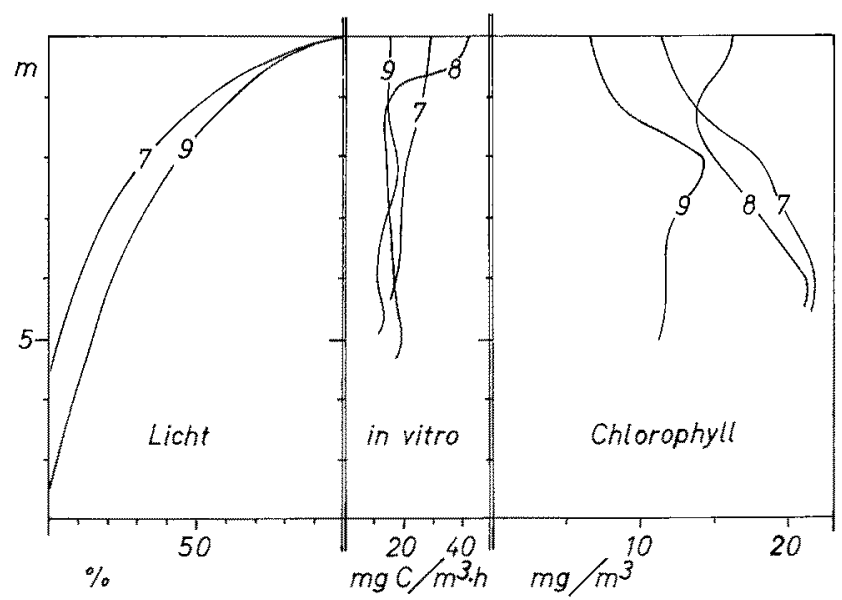

Abb. 3B: Durchschnittliche Lichtyerteilung, Photosyntheseaktivität „in vitro" im Rotationsthermostaten und Chlorophyllmengen in den verschiedenen Gewässertiefen des Plußsees 1962

unterscheiden sich in ihren chemischen, physikalischen und produktionsbiologischen Eigenschaften stark voneinander. Wie aus den Abbildungen $1 \mathrm{~B}, 2 \mathrm{~B}, 3 \mathrm{~B}$ und $4 \mathrm{~B}$ ersichtlich ist, lagen die $1 \%-\mathrm{L}$ i c h t t i e f e n im Laufe der Monate Juli bis September in den Bereichen:

Schöhsee

Schluënsee
$16,0-15,0 \mathrm{~m}$
$14,0-14,5 \mathrm{~m}$
Gr. Plöner See

Plußsee
$12,0-7,5 \mathrm{~m}$ $3,9-5,0 \mathrm{~m}$

Die durchschnittlichen Temperaturen des Epilimnions der Gewässer betrugen im gleichen Zeitraum: 


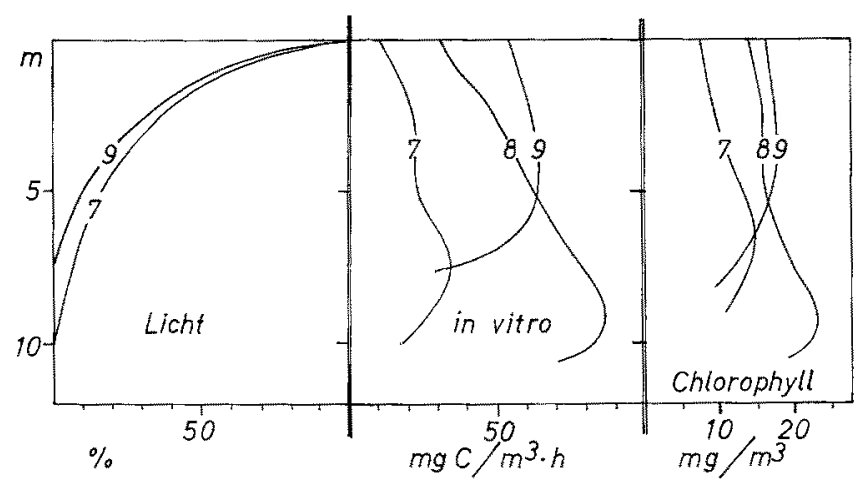

Abb. 4 B: Durchschnittliche Lichtverteilung, Photosyntheseaktivität "in vitro" im Rotationslichtthermostaten und Chlorophyllmengen in den verschiedenen Gewässertiefen des Großen Plöner Sees (Nordbecken) 1962

$\begin{array}{llll}\text { Schöhsee } & 16,2^{\circ} \mathrm{C} & \text { Plußsee } & 17,1^{\circ} \mathrm{C} \\ \text { Schluënsee } & 16,0^{\circ} \mathrm{C} & \text { Gr. Plöner See } & 15,4^{\circ} \mathrm{C}\end{array}$

Die durchschnittliche Netto-Primärproduktion des Phytoplanktons, gemessen in 24hIntervallen in ausgewählten Zonen der euphotischen Regionen, erreichte folgende Größen, die nur wenig von den in anderen Jahren festgestellten abweichen:

\begin{tabular}{|lcc|}
\hline & $\begin{array}{c}\text { Arealproduktion } \\
\mathrm{gC}^{\mathrm{m}} \mathrm{m}^{2} \times 24 \mathrm{~h}\end{array}$ & $\begin{array}{c}\text { Produktionsdichte } \\
\mathrm{mgC} / \mathrm{m}^{3} \times 24 \mathrm{~h}\end{array}$ \\
\hline Schöhsee & 0,54 & 36,8 \\
Schluënsee & 0,81 & 52,3 \\
Plußsee & 1,16 & 169,9 \\
Gr. Plöner See & 1,95 & 149,3 \\
\hline
\end{tabular}

Die Werte der Arealproduktion dieser vier Seen verhalten sich wie $1: 1,5: 2,2: 3,6$, die der Produktionsdichte jedoch wie $1: 1,4: 4,6: 4,1$. Im Plußsee entfaltet sich die photosynthetische Aktivität also auf engstem Raum, der sich aus der hoch liegenden Thermokline in diesem windgeschützt liegenden Waldsee ergibt (Abb. $3 \mathrm{C}-\mathrm{E}$ ). Eine weitere Voraussetzung ist die ständige Nährstoffversorgung des Produktionsprozesses. Fraglos wird hier der "kurzgeschlossene Stoffkreislauf" - die Mineralisation und biogene Neuverwendung der freigewordenen anorganischen Stoffe - mit sehr großer Intensität erfolgen (OHLE 1962). Entsprechend der Sedimentation von Organismen und ihren Resten, somit dem Verlust an inkorporierten Nährstoffen aus dem Epilimnion muß aber der Nährstoffnachschub auch aus den tieferen Wasserschichten beziehungsweise aus den Litoralsedimenten nicht versiegen. Tatsächlich verlaufen hier aerobe und anaerobe Abbauvorgänge außerordentlich kräftig, so daß sich im Hypolimnion riesige Mengen von Phosphat- und Ammoniumionen anreichern (Abb. $3 \mathrm{C}-\mathrm{E}$ ), die den Planktonorganismen an der Grenzschicht des Metalimnions durch Zirkulationsströmungen zugeführt werden. Dabei spielt die durch aufsteigende Schlammgase bewirkte „Methankonvektion" eine wichtige Rolle (OHLE 1959). 


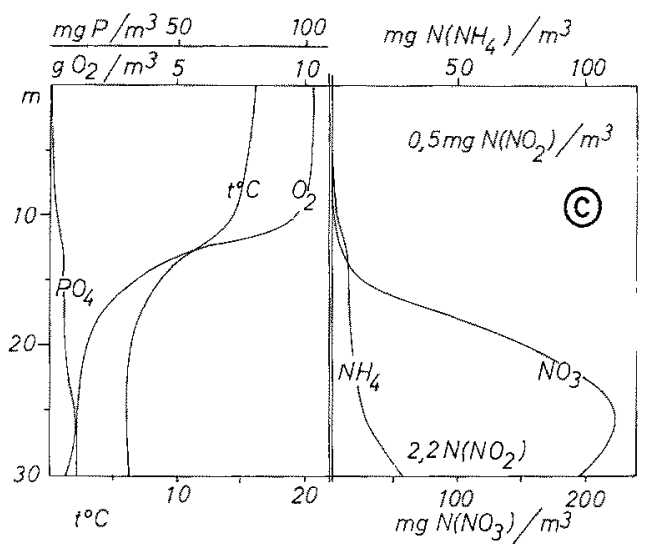

Abb. $1 \mathrm{C}$

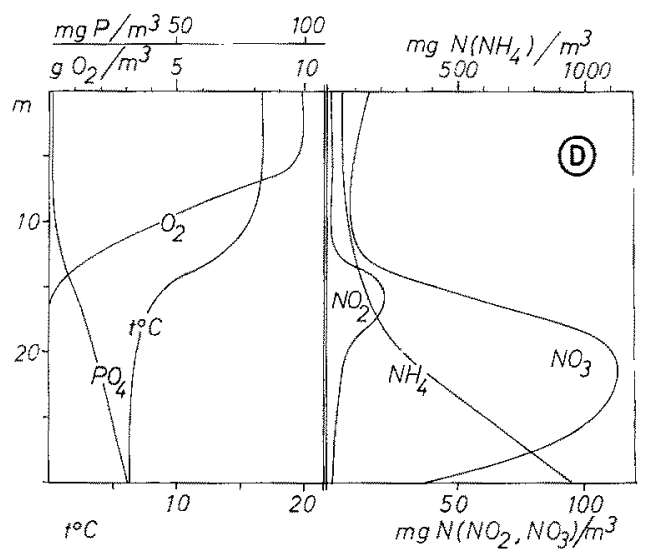

Abb. $1 \mathrm{D}$

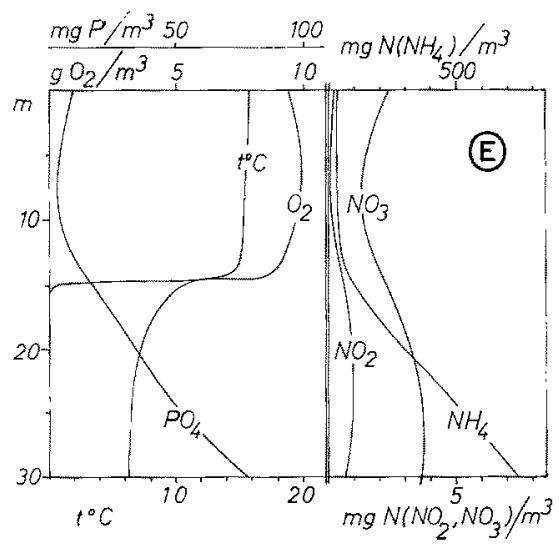

Abb. $1 \mathrm{E}$

Abb. 1 C-E: Chemische Eigenschaften der Wasserzonen des Schöhsees im Laufe des Sommers 1962 


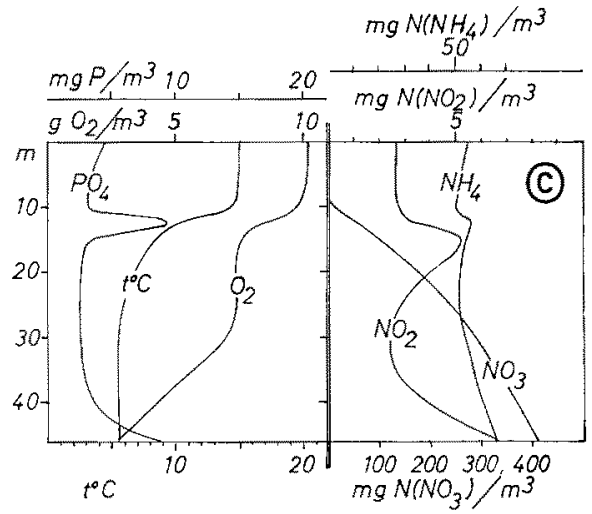

Abb. $2 \mathrm{C}$

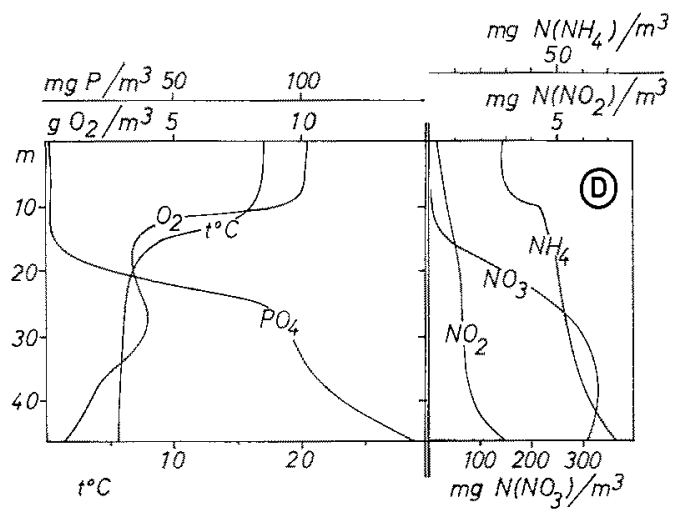

Abb. $2 \mathrm{D}$

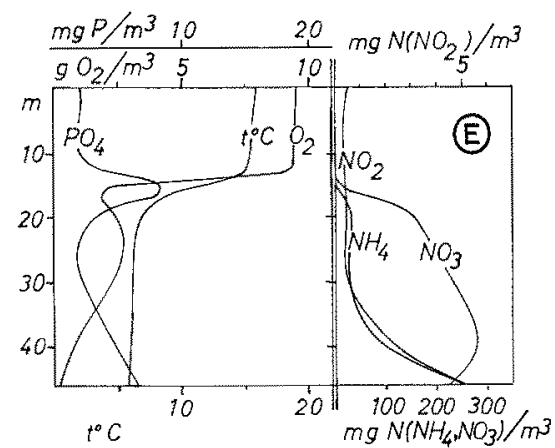

Abb. $2 \mathrm{E}$

Abb. 2C-E: Chemische Eigenschaften der Wasserzonen des Schluënsees im Laufe des Sommers 1962 


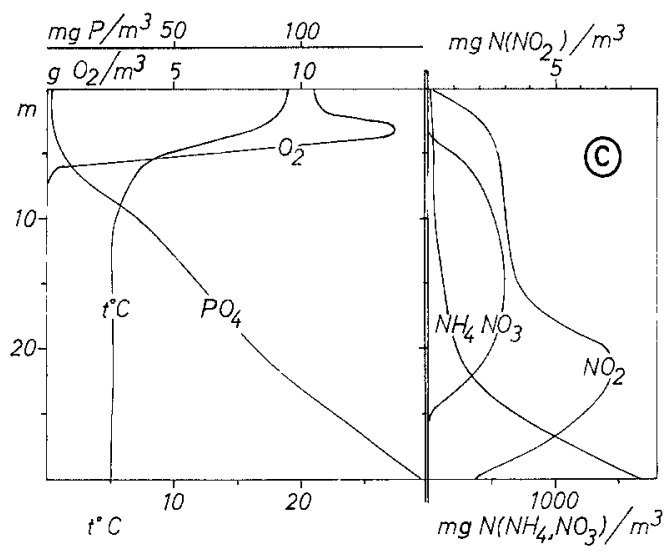

Abb. $3 \mathrm{C}$

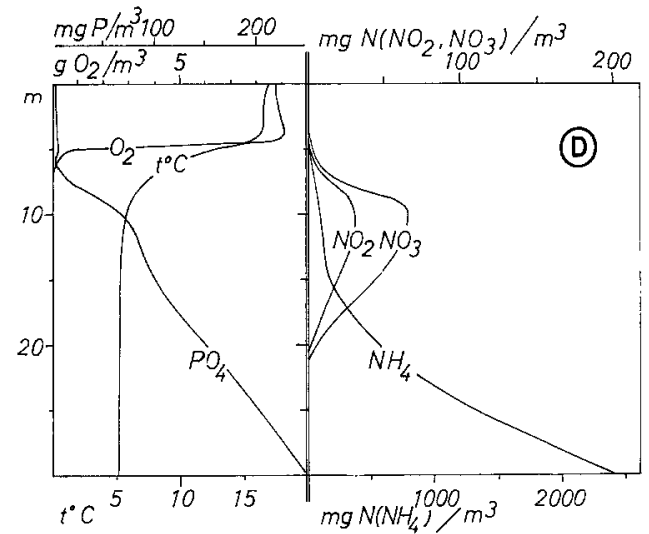

Abb. $3 \mathrm{D}$

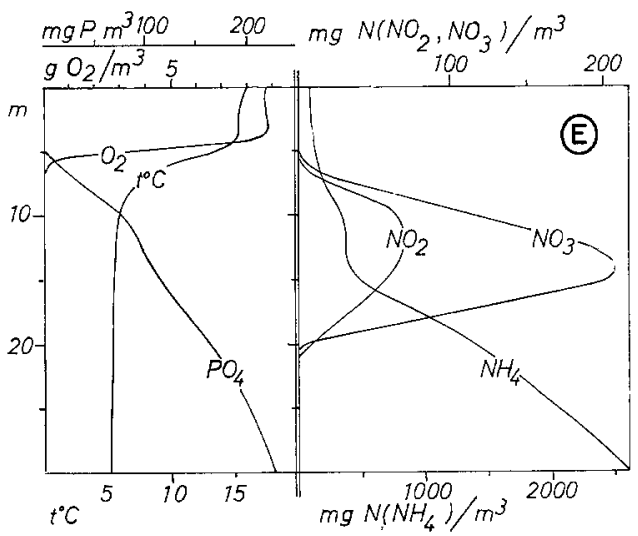

Abb. $3 \mathrm{E}$

Abb. 3 C-E: Chemische Eigenschaften der Wasserzonen des Plußsees im Laufe des Sommers 1962 


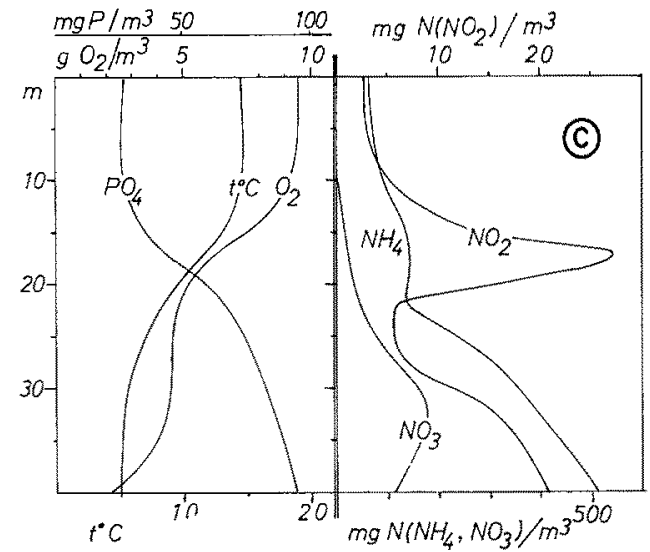

Abb. $4 \mathrm{C}$

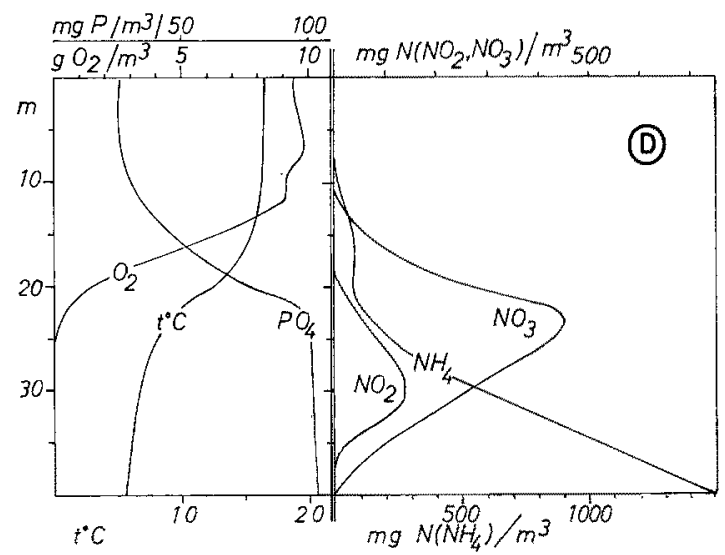

Abb. $4 \mathrm{D}$

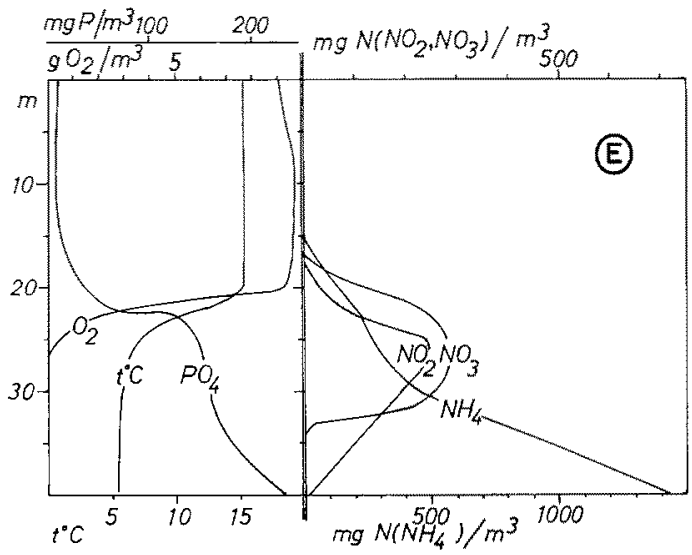

Abb. $4 \mathrm{E}$

Abb. 4 C-E: Chemische Eigenschaften der Wasserzonen des Großen Plöner Sees (Nordbecken) im Laufe des Sommers 1962 
Dieser autochthone Stoffhaushalt ist, wie unsere Untersuchungen wiederholt bestätigt haben, in den Seen viel bedeutsamer für die Primärproduktion als ein Nährstoffnachschub aus der Umgebung der Gewässer, wenigstens unter natürlichen, nicht durch Abwässer oder Erosionswässer landwirtschaftlicher Felder veränderten Bedingungen. Die Nährstoffe gelangen auf biogenem Wege zur Akkumulation in den Gewässern, werden mit den Organismenresten sedimentiert, im Schlamm oder zum großen Teil bereits beim Niedersinken, vor allem im Metalimnion, mineralisiert und erneut von den Planktonalgen aufgenommen. In Abhängigkeit von dieser Stoffwechseldynamik, in deren Ablauf die biogenen Umsetzungen der freien Wasserzonen sowohl wie der Sedimente einbezogen sind, stellt sich in einem Gewässer das Gleichgewicht der Aufbau- und Abbauprozesse ein.

Während der Hauptvegetationszeit können die für das Fortdauern der Produktion notwendigen Nährstoffreserven auf Grund der chemischen Beschaffenheit des Epilimnions selten oder gar nicht beurteilt werden (vgl. Abb. 1-4, jeweils C-E). Bis zu einem gewissen Grade wäre es möglich, die im Hypolimnion angesammelten Vorräte quantitativ darauf $\mathrm{zu}$ beziehen. Insbesondere sollten die chemischen Eigenschaften der Sedimente weitgehenden Aufschluß über ihre Bedeutung als Nährstofflieferanten geben (Tab. 1). Das ist auch überwiegend der Fall, läßt jedoch gerade hinsichtlich der Phosphor- und Stickstoffversorgung nur bedingte Aussagen zu, weil nicht die Gesamtmenge an diesen Verbindungen dem überstehenden Wasser überliefert wird, sondern ein unter dem Einfluß von Mikroben freigesetzter Teil. Er wird überdies durch hemische und kolloidchemische Prozesse reguliert, an denen Eisen-, Aluminium-, Calcium-, Silikat- und Humusverbindungen beteiligt sind (OHLE 1964).

Infolgedessen haben wir die Interstitiallösungen der Sedimente durch Zentrifugierung gewonnen und analysiert (Tab. 2), um direkte Unterlagen über die Nährstoffreserven zu erhalten, die durch Diffusion oder unter Beteiligung von Tubificiden und

Tabelle 1

Chemische Zusammensetzung der Sedimente aus dem Tiefenbecken der Seen $(4-8 \mathrm{~cm}$ Schlammtiefe) $0 / 0 /$ Trockensubstanz. Si: natriumcarbonatlösliche Kieselsäure; Hum.: Huminsäure (gemäß photometrischer Bestimmung des alkalilöslichen Anteiles); Chl.: Chlorophyll a - "Aequivalent"

\begin{tabular}{|lcccc|}
\hline & Schöhsee & Schluënsee & Plußsee & Gr. Plöner See \\
\hline anorg. Substanz & 78,2 & 89,4 & 57,1 & 86,8 \\
org. Substanz & 21,8 & 10,6 & 42,9 & 13,2 \\
organ. C & 12,9 & 5,1 & 15,7 & 9,6 \\
organ. H & 1,86 & 0,78 & 3,4 & 1,36 \\
HCl-Unlösl. & 50,9 & 53,2 & 43,5 & 49,2 \\
Freie CO2 u. CO3 & 6,30 & 12,8 & 1,05 & 12,9 \\
N (Kjeldahl) & 1,12 & 0,52 & 2,3 & 0,68 \\
P & 0,04 & 0,14 & 0,18 & 0,10 \\
Si & 0,86 & 1,88 & 0,26 & 1,18 \\
Al & 1,02 & 1,14 & 1,00 & 0,31 \\
Ca & 6,4 & 13,1 & 1,9 & 11,6 \\
Mg & 6,4 & 3,4 & 3,2 & 2,5 \\
Fe & 2,80 & 3,56 & 2,75 & 1,64 \\
Mn & 0,007 & 0,11 & 0,02 & 0,26 \\
Hum. & 1,99 & 1,41 & 6,06 & 0,93 \\
Chl. & 0,013 & 0,008 & 0,030 & 0,020 \\
\hline
\end{tabular}


Tabelle 2

Durchschnittliche Zusammensetzung der Interstitiallösungen von Seesedimenten (mg/l). Hufa: Humusfarbe (in Farbeinheiten Methylorange); $\mu \mathrm{S}_{20}$ : Spezifische Leitfähigkeit bei $20^{\circ} \mathrm{C}$

\begin{tabular}{|lcccc|}
\hline Durcbschnittswerte & Schöhsee & Schluënsee & Plußsee & Gr. Plöner See \\
aus Seetiefen & $12-27 m$ & $15-42 m$ & $6-25 m$ & $16-40 m$ \\
\hline $\mathrm{pH}$ & 7,73 & 7,82 & 7,36 & 7,73 \\
$\mathrm{Hufa}$ & 22 & 45 & 50 & 85 \\
$\mu \mathrm{S}_{20}$ & 383 & 475 & 384 & 502 \\
$\mathrm{HCO}$ & 228,8 & 257,4 & 219,0 & 356,2 \\
$\mathrm{Cl}$ & 18,5 & 35,0 & 15,0 & 52,0 \\
$\mathrm{SO}_{4}$ & 6,0 & 13,6 & 9,0 & 45,2 \\
$\mathrm{Si}\left(\mathrm{SiO}_{4}\right)$ & 7,2 & 11,4 & 7,6 & 26,8 \\
$\mathrm{Gesamt}-\mathrm{P}$ & 0,14 & 0,27 & 1,20 & 2,19 \\
$\mathrm{~K}$ jeldahl-N & 8,0 & 11,5 & 20,4 & 17,3 \\
$\mathrm{~N}(\mathrm{NH} 4)$ & 7,8 & 9,2 & 18,3 & 16,2 \\
$\mathrm{Na}$ & 13,5 & 22,8 & 10,5 & 33,0 \\
$\mathrm{~K}$ & 5,3 & 5,3 & 3,5 & 6,7 \\
$\mathrm{Ca}$ & 62,1 & 60,2 & 45,8 & 67,0 \\
$\mathrm{Mg}$ & 8,2 & 15,7 & 4,5 & 20,8 \\
$\mathrm{Fe}$ & 0,30 & 0,38 & 0,42 & 0,28 \\
$\mathrm{Al}$ & 0,03 & 0,02 & 0,008 & 0,009 \\
$\mathrm{Mn}$ & 2,03 & 4,44 & 1,36 & 2,17 \\
\hline
\end{tabular}

Chironomidenlarven, bei starker Gärung auch durch „Methankonvektion “ in das Wasser gelangen. Zunächst sei hervorgehoben, daß die Interstitiallösungen der tieferen Gewässerregionen regelmäßig bedeutend konzentrierter als die der flacheren sind. Im Nordbecken des Großen Plöner Sees wurden z. B. folgende Werte festgestellt:

\begin{tabular}{|cccc|}
\hline $\begin{array}{c}\text { Gewässertiefe } \\
\mathrm{m}\end{array}$ & $\begin{array}{c}\mathrm{HCO}_{3} \\
\mathrm{mg} / 1\end{array}$ & $\begin{array}{c}\mathrm{N}\left(\mathrm{NH}_{4}\right) \\
\mathrm{mg} / 1\end{array}$ & $\begin{array}{c}\text { Gesamtphosphat }(\mathrm{P}) \\
\mathrm{mg} / \mathrm{l}\end{array}$ \\
\hline 15 & 204 & 5,8 & 0,64 \\
18 & 298 & 8,0 & 0,87 \\
25 & 317 & 9,4 & 1,07 \\
40 & 346 & 15,3 & 2,10 \\
\hline
\end{tabular}

Für diese großen Unterschiede sind nicht nur die in der Gewässertiefe intensiver als in flacheren Gebieten erfolgenden anaeroben Abbau- und Umbauvorgänge verantwortlich zu machen, sondern auch weitere Faktoren, die sich aus der "KorngrößenFraktionierung " bei der Sedimentation anorganischer und organischer Partikel, aus der frühzeitigen, bereits während ihrer Ablagerung erfolgenden aeroben Mineralisation und im bewegten Wasser verstärkten Auswaschung sowie aus der Organismenbesiedlung des Schlammes ableiten. Jedoch soll darüber an anderer Stelle berichtet werden.

Stellt man die durchschnittlichen Konzentrationen der Interstitiallösungen an Ammonium-Ionen und Gesamt-Phosphat der vier hier als Beispiele behandelten Seen ihren Photosyntheseaktivitäten gegenüber (Abb. 5), so ergeben sich aufschlußreiche Relationen. Je größer der Ammoniumgehalt der Interstitiallösungen, um so größer ist auch die Produktionsdichte der Seen. Das gleiche Resultat ergibt sich für die Konzentrationen an Gesamt-Phosphat. Jedoch liegt sie im Plußsee so hoch, daß dadurch bereits 


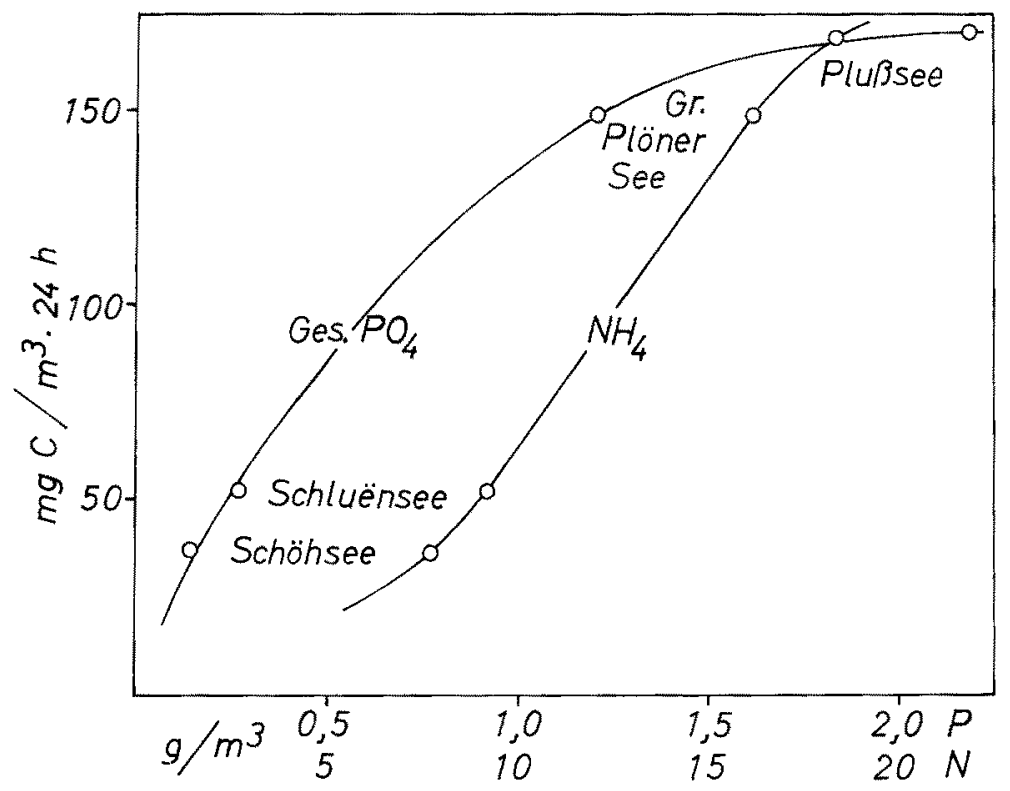

Abb. 5: Die durchschnittliche Konzentration der Interstitiallösungen an Gesamt-Phosphor und Ammonium in Beziehung zur durchschnittlichen Netto-Primärproduktion $/ \mathrm{m}^{3} \cdot 24 \mathrm{~h}$ des Schöhsees, des Schluënsees, des Plußsees und des Großen Plöner Sees im Sommer 1962

keine wesentliche Steigerung der Primärproduktion mehr ausgelöst werden kann. Vielmehr nähert sich die Kurve asymptotisch der Abszisse. Es erscheint durchaus möglich, daß die Primärproduktion durch vermehrte Stickstoffversorgung im Plußsee noch vergrößert werden könnte, es sich also um ein Stickstoffminimum handeln würde. Kulturversuche mit Addition von Ammonium, Nitrat oder Phosphat sollen künttighin darüber nähere Aufklärung verschaffen.

An Hand der Interstitiallösungen, die als ein wichtiges Medium der biogenen Stoffwechseldynamik anzusehen sind, lassen sich die Gewässer, wie gezeigt wurde, produktionsbiologisch charakterisieren. In diesem Zusammenhang bietet es sich an, die Ionenkonzentrationen, die sich im Laufe der Stagnationsperiode maximal in bodennahen Wässern als Folge des Stoffaustausches zwischen Schlamm und Wasser einstellen, mit dem Gehalt der Interstitiallösungen zu vergleichen (Tab. 3). Es ist erklärlich, daß die spezifische Leitfähigkeit der letzteren sowie ihr Bicarbonatgehalt die entspre-

Tabelle 3

Relation der Konzentrationen Bodennahes Wasser : Interstitiallösung

\begin{tabular}{|lccccc|}
\hline & $\mu \mathrm{S}_{20}$ & $\mathrm{HCO}_{3}$ & $\mathrm{Si}$ & $\mathrm{NH}_{4}$ & $\mathrm{PO}_{4}$ \\
\hline Schöhsee & 0,70 & 0,58 & 0,13 & 0,08 & 0,10 \\
Schluënsee & 0,70 & 0,56 & 0,22 & 0,12 & 0,09 \\
Plufsee & 0,55 & 0,45 & 0,15 & 0,30 & 0,32 \\
Gr. Plöner See & 0,46 & 0,41 & 0,10 & 0,11 & 0,17 \\
\hline
\end{tabular}


chenden Werte des bodennahen Wassers um 30 bis $60 \%$ übertriff. Jedoch liegen die Mengen an Phosphat, Ammonium und Silikat im Interstitialwasser um ein Vielfaches höher als im Wasser, ein Zeichen für das ausgeprägte Konzentrationsgefälle, das sich für diese Komponenten an der Grenzzone ergibt, und zwar bedingt durch den Verbrauch des Planktons in der euphotischen Gewässerzone, wohin die Nährstoffionen in der beschriebenen Weise durch Strömungen gelangen.

\section{VERTIKALZONIERUNG DER PHOTOSYNTHESEAKTIVITAT, DES PLANKTONCHLOROPHYLLS UND DER NAHHRSTOFFE}

Die Rückführung von Mineralisationsprodukten aus den Sedimenten erfolgt, wie im vorigen Kapitel ausgeführt, nicht nur im Profundal, sondern auch im Sublitoral und Litoral, teilweise hier infolge der höheren Temperaturen und entsprechender Intensivierung der aeroben Oxydation sogar mit erheblich größerer Geschwindigkeit. Auch die starken Turbulenzströmungen und windinduzierte laminare Turbulenz der Wassermassen - bis in die oberen Bereiche des Hypolimnions hinab - tragen zur Steigerung der Ionenabgabe aus den Sedimenten wesentlich bei, da sich keine Mikrozonierungen der gelösten Stoffe zwischen Schlamm und Wasser ausbilden können, die Grenzzone sich vielmehr immer durch ein starkes Konzentrationsgefälle auszeichnet. Sofern die von den Sedimenten ausgeschiedenen Nährstoffe vom Phytoplankton begierig aufgenommen werden - unter anderem Phosphat- und Ammoniumionen - sind sie infolge ihrer ephemeren Existenz, wenigstens während der Hauptvegetationszeit, auf chemischem Wege nur in Spuren oder gar nicht nachweisbar. Wohl bilden sich in euproduktiven Gewässern unter diesen Umständen metalimnische Sauerstoffminima aus, die allerdings auch andersartig bedingt sein können, oder Anreicherungen mit Kohlendioxyd etc. treten dort auf, die P- und N-Verbindungen jedoch werden durch Phytound Bakterioplankton zum größten Teil festgelegt, bevor eine metalimnische Zunahme nachweisbar wäre.

Was sich bei der chemischen Schichtungsuntersuchung der Seen vom Epilimnion bis zum Hypolimnion als Konzentrationsgefälle der Ionen abzeichnet (Abb. 1-4, C-E), stellt also ein Integral der Nährstoffzufuhr aus den Sedimenten und des biogenen Verbrauches im Wasser dar. Uberdies spielt dabei das Verhältnis des Schlammareals zum Wasservolumen eine entscheidende Rolle. Die Stoffwechseldynamik erreicht in Grenzgebiet zwischen Epilimnion und Metalimnion ihre größte Entfaltung; denn dort stehen die euphotische Produktionszone und andererseits die vom Litoral und Sublitoral durch horizontal gerichtete Wasserturbulenz herangeführten, mit Nährstoffen angereicherten Wasserkörper in engstem Kontakt miteinander (vgl. Alst'erberg 1930, Mortimer 1952).

Infolge ausreichender Nährstoffversorgung finden die Phytoplankter im unteren Teil des Epilimnions bedeutend günstigere Photosynthese-, Wachstums- und Vermehrungsbedingungen als in der obersten Gewässerregion, sobald sich hier der Nährstoffvorrat seiner Erschöpfung nähert. Die Seen erscheinen dann auffällig klar in grünblauer Färbung, ähnlich derjenigen mancher Alpenseen. Es wäre indessen ein Trugschluß, anzunehmen, es handle sich um extrem planktonarme Gewässer. Vielmehr 
halten sich die Algen dann in größerer Tiefe des Epilimnions, häufig in relativ dünner Schicht über dem Metalimnion zusammengedrängt auf, wohin nur etwa 1 bis $5 \%$ des eingestrahlten Sonnenlichtes gelangen, an sonnenklaren Tagen annähernd 500 bis 1000 Lux, wenn es erlaubt sein mag, die grüne Reststrahlung in diesen anders defi-

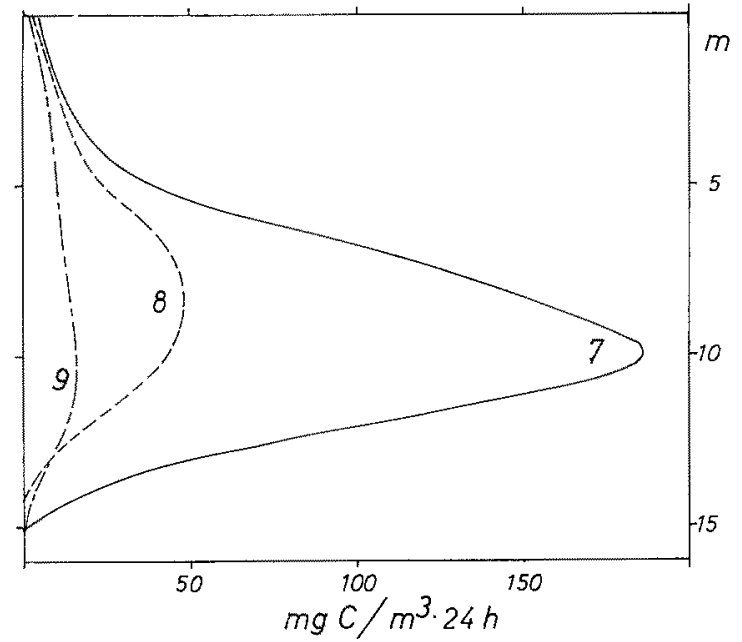

Abb. 1A: Vertikalzonierung der Primärproduktion des Schöhsees im Sommer 1962

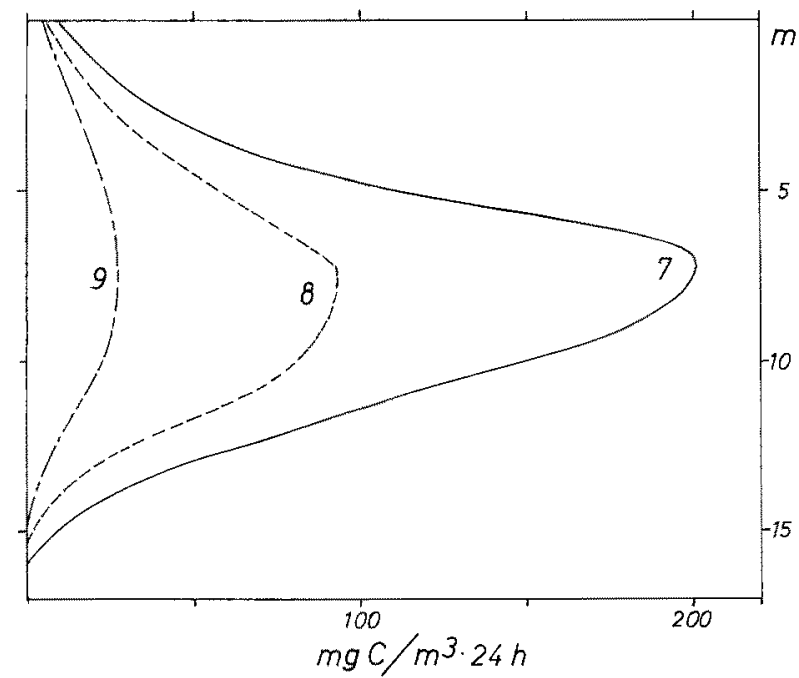

Abb. 2 A: Vertikalzonierung der Primärproduktion des Schluënsees im Sommer 1962

nierten Beleuchtungseinheiten anzugeben. So befand sich im August 1960 z. B. im Schöhsee eine Massenentwicklung von Oscillatoria Agardhii eng begrenzt in der Wasserschicht zwischen 14 und $16 \mathrm{~m}$ Tiefe, und bis $8 \mathrm{~m}$ Tiefe waren überhaupt keine Oscillatoria-Trichome aufzufinden. 
Unter diesen Gesichtspunkten geben uns die in einigen holsteinischen Gewässern häufig beobachteten, hier speziell von vier Seen wiedergegebenen konvexen Verteilungskurven keine unlösbaren Rätsel auf (Abb. 1A-4A). Es handelt sich um eine typische Optimalzonierung, die beiderseits einem Pejus beziehungsweise Pessimum zu-

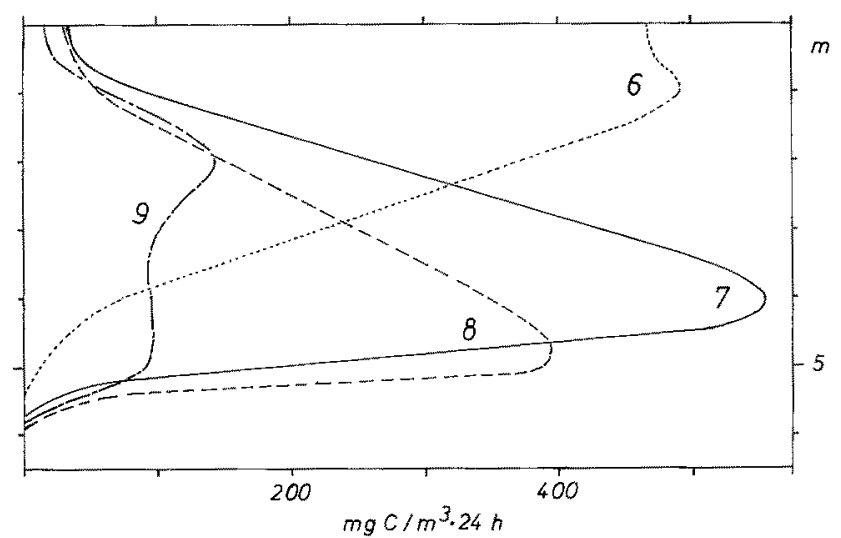

Abb. 3 A: Vertikalzonierung der Primärproduktion des Plußsees im Sommer 1962

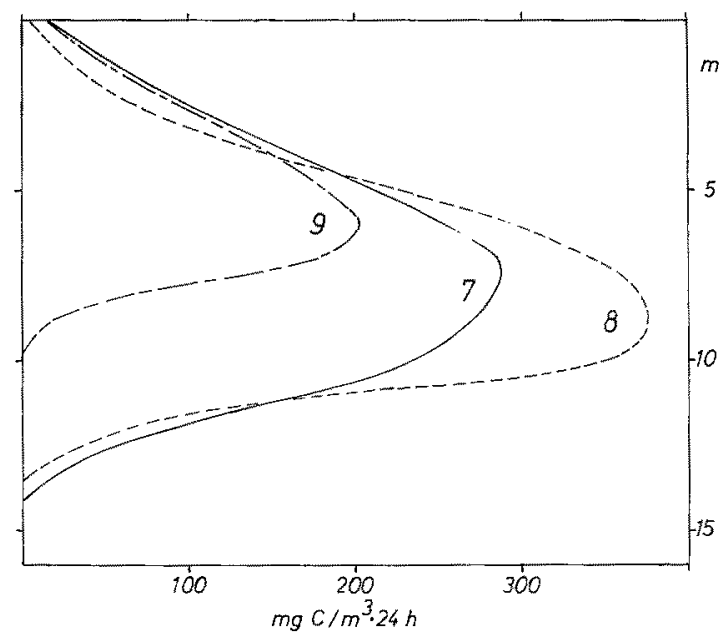

Abb. 4 A: Vertikalzonierung der Primärproduktion des Großen Plöner Sees (Nordbecken) im Sommer 1962

strebt, nach oben im Gewässer bedingt durch Nährstoffmangel (teilweise nahe der Gewässeroberfläche auch durch extrem hohe Lichtdosis), nach unten durch Lichtmangel (teilweise auch durch niedere Temperaturen).

Ahnliche, gleichfalls mit der ${ }^{14} \mathrm{C}$-Methodik bestimmte Vertikalverteilungen der Primärproduktion stellten FINDENEGg und RodHe (FINDENEGg 1959) im Oktober 1958 in Seen Kärntens und des Salzkammerguts fest, besonders deutlich ausgeprägt im Längsee, wo das Photosynthesemaximum in $10 \mathrm{~m}$ Tiefe durch Oscillatoria rubescens 
hervorgerufen wurde. Die Anhäufung der Burgunderblutalge im Tiefenbereich zwischen 8 und $15 \mathrm{~m}$ ist charakteristisch für das Gewässer, wie FindenEGg (1958) auf Grund der gemäß Rechenvolumen ermittelten Planktonbiomassen angegeben hat. Auch im Wörthersee ist Oscillatoria rubescens, die sich im Herbst in metalimnischen Zonen aufhält, für einen in 12 bis $14 \mathrm{~m}$ Tiefe beobachteten Anstieg der Aminilationsintensität verantwortlich. Im mesohumosen Lunzer Obersee dagegen wurde er nach RoDHE (Findenegg 1959) in $4 \mathrm{~m}$ Tiefe durch Gymnodinium pascheri und Peridinium aciculiferum bedingt und erreichte den riesigen Wert von $630 \mathrm{mg} \mathrm{C} / \mathrm{m}^{3} \cdot 24 \mathrm{~h}$, allerdings eng begrenzt auf eine Wasserschicht von etwa $1 \mathrm{~m}$ Mächtigkeit.

Die Einschichtung von Oscillatoria rubescens wurde desgleichen durch THomas \& MÄrKI (1949) vom Zürichsee sowie vom Baldeggersee und Hallwilersee durch BACHOFEN (1960) beschrieben, so daß auch in diesen Gewässern die stärkste Photosyntheseaktivität während der Sommerstagnation im Metalimnion oder wenig oberhalb davon auftreten wird.

In den vier hier behandelten holsteinischen Seen wurde Oscillatoria rubescens im Jahre 1962 nicht festgestellt, jedoch seit dem Februar 1963 im Schöhsee, als sich unter der langwährenden Eisdecke in großer Menge die rötlichen Flocken ansammelten. Auch in diesem Winter (1963/64) trat das gleiche Phänomen auf, und zwar außerordentlich verstärkt. Es besteht kein Zweifel darüber, daß diese "Invasion“ auf die Nährstoffanreicherung des Sees durch Abwässer und durch die seit dem letzten Sommer erfolgte Einschüttung von nährstoffreichem Boden zurückzuführen ist. Die weiterhin durchgeführten chemischen Wasseranalysen liefern die Bestätigung für die Ursache dieser Auxotrophierung. Allerdings ist zu betonen, daß Oscillatoria rubescens in früheren Jahren bereits im Schlöhsee vorhanden war (UTERMöHL 1925), späterhin infolge geringer Mengen jedoch übersehen wurde.

Das im Bereich von 8 bis $12 \mathrm{~m}$ Tiefe des Schöhsees im Sommer 1962 ständig vorliegende Photosynthesemaximum (Abb. 1A) wurde durch Oscillatoria Agardhii und Anabaena planktonica bewirkt. Die übrigen Plankter, darunter Asterionella formosa, Diatoma elongatum, Ceratium birundinella und Aphanizomenon flos-aquae, gelangten nicht zur Massenentwicklung. Nur kurzfristig machte sich Dinobryon sociale als Wasserblüte bemerkbar. Die Planktonorganismen wurden nicht gezählt, jedoch die Chlorophyllmengen photometrisch bestimmt (Abb. 1B). Die Chlorophyllmaxima befanden sich in Konzentrationen bis zu $15,5 \mathrm{mg}$ Chlorophyll $\mathrm{a} / \mathrm{m}^{3}$ in den gleichen Gewässer-

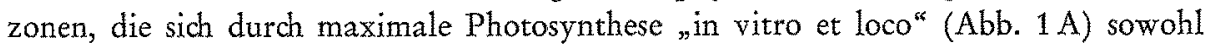
wie „in vitro" (Abb. $1 \mathrm{~B}$ ), im Juli mit annähernd $50 \mathrm{mg} \mathrm{C} / \mathrm{m}^{3} \cdot \mathrm{h}$, auszeichneten.

Oscillatoria prolifica, die O. rubescens nahe verwandte, von manchen Autoren damit vereinigte Art, ist seit einigen Jahren im Plußsee zu starker Entfaltung gelangt und sammelt sich im Winter in großen Flocken an, ebenso wie es bereits vom Schöhsee für die mit typischer kopfiger Kalyptra versehene Oscillatoria rubescens (HUBERPestalozzi 1938, 1962) angegeben wurde. Oscillatoria prolifica trat nach Angaben meines Herrn Kollegen Dr. SchWABE im Plankton des Plußsees im September 1962 auf. Die Hauptmengen des Planktons stellte jedoch Oscillatoria Redekei, insbesondere in den durch maximale Photosynthese hervortretenden Wasserschichten. Dort betrug der Chlorophyllgehalt im Juni $196222 \mathrm{mg} / \mathrm{m}^{3}$ (Abb. 3B), die 24stündige Exposition im See („in vitro et loco“) lieferte eine Primärproduktion von $560 \mathrm{mg} \mathrm{C} / \mathrm{m}^{3}$ (Abb. $3 \mathrm{~A}$ ), 
und der Wert im Rotationslichtthermostaten war nur $37 \mathrm{mg} \mathrm{C} / \mathrm{m}^{3} \cdot \mathrm{h}$ (Abb. 3B). Es kann aus diesem Befund der Schluß gezogen werden, daß die Lichtintensität von 4400 Lux, der die Wasserproben im "Rolitherm" ausgesetzt wurden, bereits überoptimal war für die im See an geringere Lichtexposition gewöhnten Algen. Tatsächlich gelangten die Oscillatorien und begleitenden Algen im August 1962 in $9 \mathrm{~m}$ Tiefe des Plußsees zu maximaler Photosynthese bei nur 400 Lux. Es handelte sich im Sinne von SteEmann Nielsen (1961) und Ryther (1959) also um „Schattenpflanzen“. Staub (1961) stellte bei seinen autökologischen Untersuchungen von Oscillatoria rubescens fest, wie hier vergleichsweise erwähnt sei, daß diese Cyanophycee bei mehr als 2000 Lux gehemmt wird, bei 1500 Lux aber voll lebensfähig ist. Von den Planktonarten des Plußsees sind außerdem hervorzuheben: Aphanizomenon gracile (vgl. SCHWABE \& Stange-Bursche 1964), Anabaena flos-aquae spiroides, Tetraedon minimum, Ceraticum birundinella, Asterionella formosa, Centronella reichelti und Stephanodiscus bantzschi.

Oscillatorien haben sich auch im Großen Plöner See mehr und mehr zu Hauptvertretern im sommerlichen Plankton entwickelt. In großen Mengen traten 1962 Oscillatoria Redekei und O. Agardhii auf, die an der Ausbildung der konvexen Produktionskurven wesentlich beteiligt waren (Abb. 4A). Auch Anabaena flos-aquae spiroides trat häufig auf, außerdem Diatoma elongatum, Ceratium birundinella und Microcystis bolsatica. Den Tiefenlagen der Photosynthese-Maxima (Abb. $4 \mathrm{~A}$ ) entsprachen die maximalen Chlorophyllmengen (Abb. 4B). Es waren im August in $9 \mathrm{~m}$ Tiefe $24 \mathrm{mg}$ Chlorophyll a/m $\mathrm{m}^{3}$, und die Primärproduktion erreichte dort $380 \mathrm{mg}$ $\mathrm{C} / \mathrm{m}^{3} \cdot 24 \mathrm{~h}$. Im Rolitherm wurden bei gleicher Temperatur, aber $4400 \mathrm{Lux}, 86 \mathrm{mg}$ $\mathrm{C} / \mathrm{m}^{3} \cdot \mathrm{h}$ gemessen. In diesem Falle und auch bei den übrigen Proben aus dem Großen Plöner See war keine Lichthemmung im Rolitherm festzustellen, sondern im Gegenteil eine Förderung der Photosynthese, die, verglichen mit den Originalbedingungen der Proben, auf der wesentlich stärkeren Beleuchtung beruhte.

Als gänzlich frei von planktischen Oscillatorien erwies sich bislang der Schluënsee. Die Primärproduktion, auch hier im Laufe des Sommers mit der ausgeprägten Vertikalzonierung, die ebenso wie bei den drei anderen Gewässern vom Lichtgefälle abwich, erfolgte vorwiegend durch Anabaena flos aquae spiroides, Ceratium birundinella, Asterionella formosa, Scenedesmus quadricauda und Microcystis aeruginosa. Das Maximum der Photosynthese wurde im Juli in $7 \mathrm{~m}$ Tiefe festgestellt und betrug $202 \mathrm{mg}$ $\mathrm{C} / \mathrm{m}^{3} \cdot 24 \mathrm{~h}$. Der Chlorophyllgehalt dieser Region war $19 \mathrm{mg} \mathrm{C} / \mathrm{m}^{3}$, und , in vitro“ wurde der Wert $21 \mathrm{mg} \mathrm{C} / \mathrm{m}^{3}$ bestimmt.

Vergleicht man die Primärproduktion der vier Seen der Monate Juli bis September miteinander (Abb. $1 \mathrm{~A}$ bis $4 \mathrm{~A}$ ), so fällt auf, daß mit Ausnahme des Großen Plöner Sees ein stetiger Rückgang erfolgte. Gleichzeitig verringerten sich die Phosphat-, Nitrat- und Ammonium-Konzentrationen in der euphotischen Zone. Im Großen Plöner See jedoch blieben die Phosphatmengen des Epilimnions nahezu konstant. Es ist anzunchmen, daß es sich um eine direkte Folge der recht erheblichen Abwassereinleitung handelt, die ja auf Grund der tippigen Verwendung von phosphatreichen Waschmitteln im Haushalt mit starken Phosphatzufuhren verbunden ist. So erklärt sich die im August gegenüber dem Juli im Großen Plöner See zum Unterschied von den anderen Gewässern noch ansteigende Primärproduktion. Erst im September reicht auch diese 
zivilisatorische Phosphatanreicherung nicht mehr für den chemaligen Produktionsumfang aus.

Die Primärproduktion des Phytoplanktons bleibr, wie zusammenfassend festzustellen ist, in starkem Maße vom Nährstoffnachschub abhängig und damit, wie im vorhergehenden Kapitel näher beleuchtet wurde, von den Gewässersedimenten mit ihren Organismen. Die Faktoren Licht und Nährstoffe, insbesondere Phosphor- und Stickstoffverbindungen, da es an anderen "biogenen Elementen" in den holsteinischen Gewässern kaum jemals mangelt, bestimmen den Habitus der Photosyntheseintensität in ihrer vertikalen Ausbreitung und regulieren den gesamten Umfang der Primärproduktion. Da aber die Lichtversorgung für diese Seen als "konstanter" Faktor einzusetzen ist, stellen die Phosphat- und Ammoniumkonzentrationen der Interstitiallösungen des Schlammes die entscheidende Basis der biogenen Stoffwechseldynamik dar. Die qualitative Zusammensetzung der Interstitiallösungen ebenso wie ihre Konzentrationen an Ionen sind von der Gegenwart anorganischer und organischer Kolloide des Schlammes und nicht zuletzt von der Biomasse und Bioaktivität der Bakterien (RoDina 1958) abhängig. Diese Prozesse gehören daher zur wichtigen experimentellen Aufgabe unserer weiteren Untersuchungen.

\section{ZUSAMMENFASSUNG}

1. Die Intensitäten der Photosynthese des Schöhsees, Schluënsees, Plußsees und GroBen Plöner Sees unterscheiden sich auf Grund ihrer Nährstoffreserven, insbesondere an Phosphor- und Stickstoffverbindungen, wesentlich voneinander.

2. Das Nährstoffreservoir stellen die Interstitiallösungen des Schlammes dar, die mit ihrem Gehalt an Gesamt-Phosphor und Ammoniumionen in direkter Relation zur Primärproduktion des Phytoplanktons $/ \mathrm{m}^{3} \times 24 \mathrm{~h}$ der euphotischen Gewässerzonen stehen.

3. Im Laufe des Sommers (1962) verringert sich die Primärproduktion der Seen gleichlaufend mit der Erschöpfung der Phosphat- und Stickstoffverbindungen im Epilimnion. Im Großen Plöner See tritt dieser Rückgang der Primärproduktion infolge zivilisatorisch bedingter Phosphatzufuhr jedoch später als in den anderen drei hier behandelten Gewässern ein.

4. Alle vier Seen zeichnen sich während der sommerlichen Schichtungsperiode (1962) ständig durch eine ausgeprägte Vertikalzonierung der Primärproduktion mit Maximalwerten im unteren Bereich des Epilimnions aus.

5. Diese Vertikalverteilung ist zurückzuführen erstens auf die Adaptation der Algen (vor allem von Oscillatoria- und Anabaena-Arten) an schwache Beleuchtungsintensitäten, zweitens auf die günstige Nährstoffversorgung am Grenzgebiet des Metalimnions, insbesondere bewirkt durch laminare Turbulenzströmungen der Wassermassen aus den Interstitiallösungen des Sublitorals.

Die Untersuchungen wurden mit Unterstützung der Deutschen Forschungsgemeinschaft durchgeführt. 


\section{ZITIERTE LITERATUR}

Austerberg, G., 1930. Die Ausgleichströme in den Seen im Sommerhalbjahr bei Abwesenheit der Windrichtung. Int. Rev. Hydrobiol. Hydrogr. 25, 1-32.

BACHOfEN, R., 1960. Stoffhaushalt und Sedimentation im Baldegger- und Hallwilersee. Diss. Zürich, $118 \mathrm{pp}$.

FindeneGG, J., 1959. Die Gewässer Osterreichs. Ein limnologischer Überblick. Biol. Stat. Lunz, 14. Int. Limnol. Kongr. (Spec. publ.), 86 pp.

- 1958. Trophiezustand und Produktion der Kärntner Seen. Verh. int. Ver. Limnol. 13, $170-180$.

GessNer, Fr, 1959. Hydrobotanik Bd. 2: Stoffhaushalt. VEB Dt. Verl. d. Wiss., Berlin, 701 pp.

Huber-Pestalozzi, G., 1938; 1962. Das Phytoplankton des Süßwassers. In: Die Binnengewässer. Bd. 16, T. 1. Schweizerbart, Stuttgart, 342 pp.

Mor Trmer, C. H., 1952. Water movements in lakes during summer stratification: Evidence from the distribution of temperature in Windermere. Pbil. Trans. (B) 236, 355-404.

Mulin, J. B. \& Riley J. P., 1955. The spectrophotometric determination of nitrate in natural waters, with particular reference to sea water. Analytica chim. Acta 12, 464-480.

Ofru, W., 1953. Phosphor als Initialfaktor der Gewässereutrophierung. Jb. Wasser 20, 11-23.

- 1959. Die Stoff wechseldynamik der Seen in Abhängigkeit von der Gasausscheidung ihres Schlammes. Jb. Wasser 25, 127-149.

- 1962. Der Stoffhaushalt der Seen als Grundlage einer allgemeinen Stoff wechseldynamilk der Gewässer. Kieler Meeresforsch. 18, 107-120.

- 1964. Kolloidkomplexe als Kationen- und Anionenaustauscher in Binnengewässern. $J b$. Wasser 30, 50-64.

RodHe, W., Vollenweider, R. A. \& NAUwerCK, A., 1956. The primary production and standing crop of phytoplankton. In: Perspectives of marine biology. Ed. by A. A. BuzzatiTraverso. Sympos. Scripps Inst. Oceanogr., Univ. of Calif. pr., Berkeley, Calif., 229-322.

Rodrna, A. G., 1958. Mikroorganismen und die Erhöhung der Produktivität von Teichen. Verl. Akad. Wiss. USSR Moskau, Leningrad, $170 \mathrm{pp}$.

Ryther, J. H., 1956. Photosynthesis in the ocean as a function of light intensity. Limnol. Oceanogr. 1, 61-70.

- \& MenzeL, D. W., 1959. Light adaptation by marine phytoplankton. Limnol. Oceanogr, 4, 492-497.

Schwabe, G. H. \& Stange-Bursche, Eva-M, 1964. Uber Apbanizomenon gracile Lemm. (Blaualgen und Lebensraum. 9.). Arch. Hydrobiol. 60, 249-277.

STAUB, R., 1961. Ernährungsphysiologisch-autökologische Untersudhungen an der planktischen Blaualge (Oscillatoria rubescens DC.). Schweiz. Z. Hydrol. 23, 82-198.

Steemann Nielsen, E., 1952. On detrimental effects of high light intensities on the photosynthetic mechanism. Pbysiol. Plant. 5, 334-344.

- \& Hansen, V. K., 1959. Measurements with the carbon-14 technique of the respiration rates in natural populations of phytoplankton. Deep Sed Res. 5, 222-223.

- 1961. Influence of surface illumination on plankton photosynthesis in Danish waters $\left(56^{\circ} \mathrm{N}\right)$ throughout the year. Pbysiol. Plant. 14, 595-613

- \& Jensen, E. A., 1957. Primary oceanic production. The autotrophic production of organic matter in the oceans. Galatbea Report 1, 49-136.

Talling, J. F., 1957. Photosynthetic characteristics of some freshwater plankton diatoms in relation to underwater radiation. New Phytol. 56, 29-50.

Thomas, E. A. \& MärkI, E., 1949. Der heutige Zustand des Zürichsees. Verh. int. Ver. Limnol. 10, 476-488.

UnGEMACH, H., 1960. Sedimentchemismus und seine Beziehungen zum Stoffhaushalt in 40 europäischen Seen. Diss. Kiel (Ms), 420 pp.

UTERmöHL, H, 1925. Limnologische Phytoplanktonstudien. Die Besiedelung ostholsteinischer Seen mit Schwebpflanzen. Arch. Hydrobiol. Suppl. 5, 1-526.

Vollenweider, R., 1950. Okologische Untersuchungen von planktischen Algen auf experimenteller Grundlage. Schweiz. Z. Hydrol. 12, 194-262. 